\title{
Obstetric Emergencies in the Field
}

\author{
C Cox, JRA Duckett
}

\section{Introduction}

Medical Services personnel may be called upon to deal with obstetric emergencies in the pre-hospital situation or in the field hospital. Dealing competently and confidently with normal childbirth let alone obstetric emergencies is a great recruiter of 'Hearts and Minds'.

Remember, however 'Primum non nocere' (First do no harm!)

Most babies deliver without medical assistance but it should be borne in mind that 1 in 16 women in sub Saharan Africa will die as a result of childbirth and 16,000 women die each day world-wide from complications of childbirth. In addition $8,000,000$ babies a year are stillborn or die shortly after birth and many women will sustain long term damage. As Professor Hugh Maclaren said: "nature has no respect for women -she tears great holes in them". Obstetric emergencies develop quickly and dramatically. Complications include bleeding, which may be impressively profuse and epileptiform convulsions, both requiring urgent resuscitation and treatment.

"There are but two things which have an effect on me in a labour; haemorrhage and convulsions". William Hunter.

The non-specialist may make a tremendous difference to the outcome of childbirth by applying small amounts of knowledge and plenty of common sense.

\section{Management of a normal delivery}

The baby should be delivered by maternal effort unless there is a long delay between the delivery of the baby's head and the rest of the baby. If this does occur the birth canal can be opened up by flexing the mother's hips onto her abdomen (the McRobert's manoeuvre). Delivery may then be achieved by pushing the baby's head downwards, with a hand on either side of the face which will encourage delivery of the baby's anterior shoulder. This manoeuvre must be carried out during a uterine contraction. An episiotomy can be performed to widen the birth canal but may produce considerable haemorrhage if it is not promptly repaired. An episiotomy should be cut starting in the midpoint of the vulva posteriorly extending posterolaterally and avoiding the anal sphincter.

\section{Abnormal deliveries}

The common forms of abnormal deliveries are breech deliveries and twins. It is useful to remember the old midwives adages 'Hands off the breech' and 'Never turn your back on the breech'. "Hands off the breech" means that during delivery the baby should not be pulled upon as this may cause the head and arms to extend with the risk of the head becoming stuck and the baby succumbing to asphyxia. "Never turn your back on the breech" refers to the propensity of the mother to push the head out while the attendant's attention has been distracted with the result that the baby falls to the floor. The baby will have been hanging from the pelvis awaiting the next contraction. If the head of a breech baby gets stuck it is reasonable to press on the mother's abdomen suprapubically in order to flex the baby's head and push it into the pelvis. Pressure should not be applied to the fundus of the uterus. Once the nape of the baby's neck is seen the feet are grasped and lifted up and swung up over the mother's abdomen. The head of the baby should then deliver. The head should be delivered in a controlled manner to avoid sudden changes in intra cranial pressure which may lead to intra cranial bleeding. Delivery may be aided by inserting a finger into the baby's mouth and further flexing the head.

Twins occur in about one in 80 pregnancies in caucasians but twice as frequently in people of African descent. After the delivery of the first twin it is worth checking the position of the second twin. In fact after the delivery of any baby it is worth checking that there is not another baby in utero. If the baby is lying longitudinally there is no problem but if the baby is lying obliquely or transverse then an attempt should be made to turn the baby either head down or breech first. Contractions should then be awaited. It may be necessary to maintain the position of the baby by abdominal palpation. With twins all the complications of pregnancy are increased, especially post partum haemorrhage.

\section{Following Delivery}

Once the baby has delivered it should be dried. This will often stimulate respiration. The baby should be kept warm. Placing the baby to the mother's breast to suckle leads to the release of maternal oxytocin and
Medway Maritime

Hospital, Gillingham 
encourages delivery of the placenta by causing the uterus to contract. In the field situation it is probably best to await spontaneous delivery of the placenta. More experienced people may employ the use of oxytocic drugs given at the time of delivery of the anterior shoulder of the baby, in conjunction with controlled traction on the umbilical cord. However, too much traction may lead to the cord snapping with the risk of fetal exsanguination.

There is no hurry to ligate the cord if the placenta has not delivered. However, if the cord has snapped the end of the cord nearest the baby should be ligated no nearer than $5 \mathrm{cms}$ from the baby (as long as there is sufficient cord). When cord pulsations have ceased the cord may be ligated.

The placenta and the membranes should be inspected to check that they appear complete. The perineum should be checked for damage or bleeding. Uncomplicated tears of the perineum, which are not bleeding, do not require to be repaired. The perineum should however be examined for evidence of arterial bleeding and for damage to the anal sphincter and the urethral area.

\section{Emergencies during pregnancy and childbirth}

Two patients are at stake. In developed countries, the unborn baby is afforded considerable priority and most women would not think twice about submitting to a Caesarean section if they thought it would improve the outlook for their child.

In developing countries, however, decisions regarding management of obstetric problems are governed by the resources available at the time and which are likely to be available to the woman in the future. In many countries surgical facilities to carry out Caesarean section may be patchy and a woman who is subjected to a Caesarean section which is not performed as a life saving procedure will be left with a scar in her uterus. This poses a significant risk of scar rupture and maternal and fetal death in subsequent pregnancy.

Therefore, in these situations, every effort should be made to avoid this potentially long-term maternal-life threatening procedure. The feasibility of carrying out obstetric operations and procedures will be limited by the experience of the medical attendant and the facilities and equipment available. Once again it is important to remember the most important rule of medicine 'first do no harm'. In the majority of cases the welfare of the baby will, at best, be of secondary consideration.

The two most common life threatening emergencies occurring in pregnancy are haemorrhage and eclampsia (convulsions) or severe pre-eclampsia (toxaemia of pregnancy). Other conditions such as sickle cell anaemia are more likely to cause problems in the pregnant patient than the non-pregnant patient.

\section{Eclampsia and pre-eclampsia}

Toxaemia of pregnancy is characterized by fitting, swelling, especially around the face and eyes, the finding of protein in the urine and high blood pressure. The woman may complain of headache, flashing lights and epigastric or right hypochondrial pain thought to be due to pressure on the liver capsule as a result either of liver congestion or sub-capsular haematoma formation. In addition, the woman may pass very small quantities of urine which may be very concentrated (so called "Coca-Cola urine").

In the emergency setting, the management of the fitting mother is to protect her airway and to wait until the fit has stopped. It may then be prudent to administer anticonvulsants. Commonly available drugs, which may be useful in the control of fits include diazepam, magnesium sulphate and phenytoin, magnesium sulphate being the preferred option if it is available. The dosage of Magnesium Sulphate is $4 \mathrm{mgs}$ as a loading dose followed by a continuous infusion of $1 \mathrm{~g}$ per hour $(5 \mathrm{~g}$ in $200 \mathrm{ml}$ of normal saline over 4 hours). Recurrent seizures are treated with a further bolus of 2mgs. Opiates and promazine may be useful in the absence of the above.

The definitive treatment of eclampsia or severe pre-eclampsia is to deliver the baby, which will present problems if maternity facilities are not available! It is however very important to recognize the condition so that specialist advice may be sought.

\section{Haemorrhage during pregnancy}

Haemorrhage occurring in pregnancy is most often from the site of the placenta and is almost always maternal blood. It may come from a normally sited placenta which has separated from the wall of the uterus (placental abruption). This accounts for one third of cases. In another one third of the cases, it comes from a placenta which is attached over the cervix (placenta praevia). It is therefore essential that in cases of vaginal bleeding in pregnancy that a vaginal examination is not carried out until placenta praevia has been definitively excluded as the examination may precipitate profuse bleeding.

The remaining third of cases of vaginal bleeding are due to other causes such as bleeding after intercourse from a cervical erosion or a polyp, and occasionally infections although usually no obvious cause is found and the bleeding is not of a significant amount.

Bleeding from a significant placental abruption is almost always associated with pain and tenderness over the uterus and the mother is not infrequently clinically shocked. The baby is often dead. Delivery of the baby 


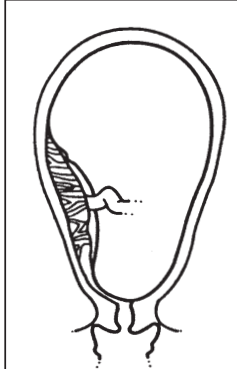

(a) Type I

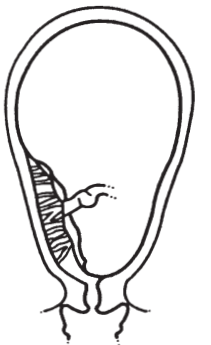

(a) Type II
Lateral

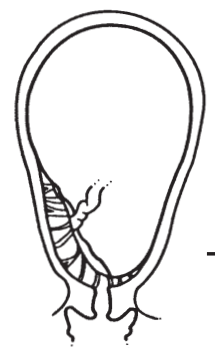

(c) Type III
Marginal

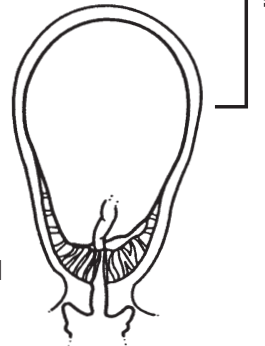

(d) Type IV

Fig 1. Placenta Praevia

should be achieved as soon as possible as problems with blood clotting and massive, often life threatening, haemorrhage frequently occur.

A vaginal examination should not be carried out in cases of suspected placenta praevia as this may well precipitate massive vaginal bleeding.

Bleeding from placenta praevia is, on the other hand, classically painless and the uterus is non tender. The baby is usually still alive despite there often being quite considerable haemorrhage. The diagnosis is confirmed by ultrasound which is increasingly available in the field as long as the necessary expertise is also available, or by a vaginal examination carried out in an operating theatre with the ability to carry out an immediate Caesarean section. This condition carries a very high maternal mortality in the absence of obstetric facilities, as the treatment is Caesarean section to deliver the baby and the abnormally situated placenta.

Sepsis is more common in women who have pre-existing anaemia or chronic

infection and in those women who have long difficult labours. If the baby has died or there have been retained products of conception after delivery the risk is further increased.

Haemorrhage may be sudden and severe after childbirth. Many women will already be anaemic and be particularly vulnerable to further blood loss, especially those women who have had bleeding prior to delivery. (It is the ante partum haemorrhage, which weakens and the postpartum haemorrhage that kills).

Bleeding can occur before, during or after delivery. If bleeding occurs before or during labour a wait and see policy should be adopted. If labour has advanced to the second stage, low forceps delivery may be carried out if someone of sufficient experience is available.

\section{Post partum haemorrhage}

Bleeding after delivery of the baby (postpartum haemorrhage) may be profuse. Has the afterbirth (placenta) delivered? Midwives and medical personnel will usually be familiar with 'controlled cord traction' this involves gentle traction on the cord at the same time lifting the uterus in the other direction towards the patients head. If this is unsuccessful and bleeding continues, a gloved hand should be introduced into the vagina to see if the placenta is sitting in the cervix. If so the placenta may be grasped and removed. If it is not, and bleeding is continuing a contraction should be "rubbed up". If the placenta does not deliver, an attempt should be made to deliver it by manual removal. This procedure requires adequate analgesia or anaesthesia and involves the gloved hand being passed into the uterus to separate the placenta from the wall of the uterus. The uterus is steadied by the other hand controlling it from above. The vaginal hand passes around the placenta in the plane between the placenta and the uterus and when the placenta has separated, it is removed from the uterus. The uterus is then explored to check that there is no retained placenta. Blood clots should be removed, an

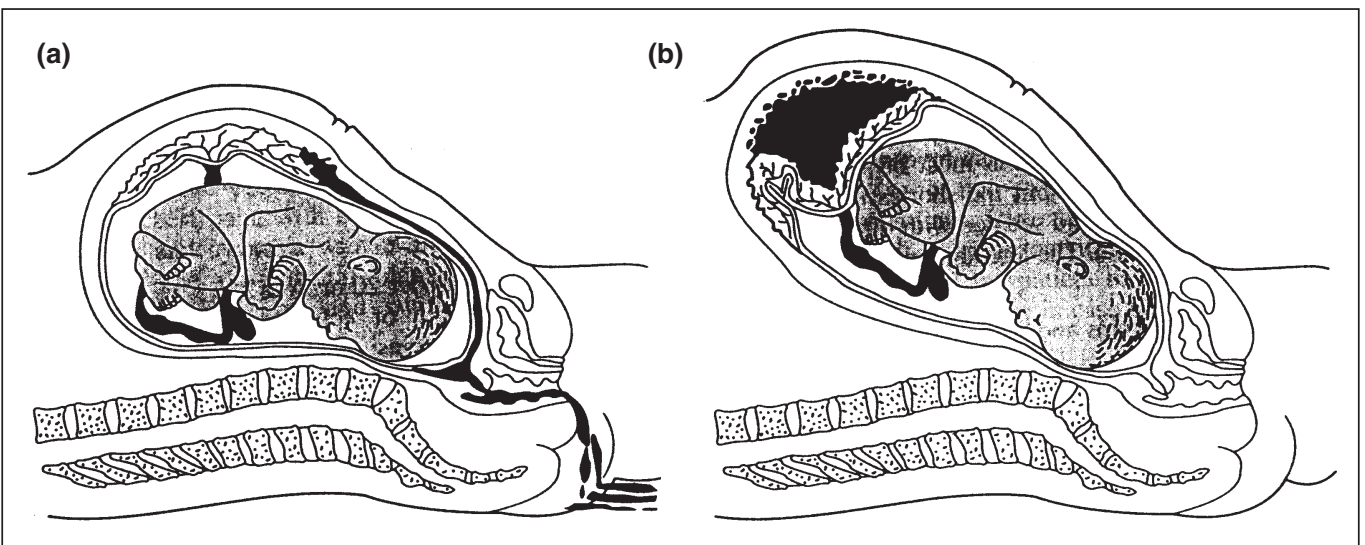

Fig 2. Placental abruption a) with revealed haemorrhage, $b$ ) with concealed haemorrhage 
oxytocic agent given and a contraction rubbed up.

An uncommon but potentially fatal problem is inversion of the uterus, such that the uterus turns inside out. The placenta may or may not be still attached to the uterus. This results in haemorrhage and collapse. If this condition is diagnosed then the prolapsed fundus of the uterus should be immediately forcibly replaced, if the placenta is still attached no attempt should be made to remove it until the inversion has been dealt with.

The commonest cause of post partum haemorrhage is relaxation (atony) of the uterus after delivery. This is treated by the use of oxytocic agents which may be repeated (usually syntometrine one ampoule). Pressure should be applied by rubbing up a contraction and the uterus should be confirmed to be empty. The empty uterus does not bleed but the uterus full of blood clot will. When the uterus is empty the uterine muscle contracts, kinks the blood vessels in the uterine wall and arrests haemorrhage (the living ligatures of the uterus).

If bleeding continues pressure can be applied to the uterus by placing a fist in the vagina, placing a hand abdominally on the back wall of the uterus and compressing the uterus between the two hands.

Bleeding may be life threatening from vaginal and vulval lacerations following delivery. This is much more common in women who have undergone circumcision (Female Genital Mutilation). In these women a 'reverse' episiotomy may be necessary to allow delivery and haemostasis will need to be achieved to control the bleeding.

It is very important to identify the upper limit to any laceration in the vagina and adequate analgisia or anaesthesia should be obtained to achieve this. Vicryl ${ }^{\circledR}$ of Dexon ${ }^{\circledR}$ are the preferred suture materials if available. The vaginal skin is closed with a continuous suture, the muscle layer in the perineum is closed with interrupted sutures making sure to take good bites of tissue and not to tie them too tight. Ideally, the perineal skin should be closed with a subcuticular stitch in order to reduce subsequent pain.

Less commonly bleeding may be from the cervix. This can be identified by using two sponge holding forceps to grasp the cervix alternately around the 360 degrees of the cervix until the bleeding point is identified and controlled with a suture.

\section{Obstructed Labour}

Women may have been in obstructed labour for several days before they present for medical help. The baby will usually be dead and the fetal tissues will then soften so that a macerated infant will usually deliver spontaneously or with the assistance of forceps. Obstructed labout in the third world is a potent cause of maternal mortality and morbidity. It can lead to infection, haemorrhage and long term damage to the bladder and the bowel resulting in loss of bladder and bowel tissue with fistula formation. This can result in leakage of urine and faeces through the vagina and consequent ostracisation of the woman.

Treatment consists of aiding delivery without causing further damage to the woman, dealing with infection and the reduction of long-term morbidity, for example by the use of indwelling urinary catheters in order to reduce the risk of fistula formation.

There may be a place for the operation of symphysiotomy if obstructed labour occurs with a live baby or under some circumstances a dead one. The procedure is comparatively simple and involves the partial division of the ligament of the symphysis pubis using an anterior approach making sure that the urethra is displaced laterally to avoid damage if the knife goes too far. This opens up the symphysis by another centimetre or so and will usually allow vaginal delivery. The patient is rested for a day or so then mobilised until she is able to climb on to a bus!

Obstructed labour in the woman who has delivered vaginally before is particularly dangerous situation as there is a high risk of uterine rupture with subsequent death of the mother and the baby. This situation requires experienced advice and may best be managed as above by a skilled operator.

\section{Post partum sepsis}

This was once a very common cause of maternal death in developed countries as 'puerperal sepsis or child-birth fever'. It is commonly caused by $\mathrm{B}-$ haemolytic streptococcus. This is an organism which is commonly present in the female vagina and is of no significance in the non pregnant. Treatment of post partum pyrexia is with a broad spectrum antibiotic such as Augmentin which covers anaerobic as well as aerobic organisms. Sepsis may cause increased loss of lochia (the normal material passed after childbirth) and it should be borne in mind that retained placental material is a potent cause of puerperal sepsis and haemorrhage. (The empty uterus does not bleed- generally!).

Breast abscesses are not uncommon and are treated aggressively with antibiotics which should cover staphlococci. If they go on to form a discrete abscess, they should be drained.

\section{Conclusion}

Pregnancy and labour although natural states nevertheless carry a significant 
mortality and morbidity both to mother and baby. In most cases a hands-off approach is best but all doctors likely to be charged with caring for women should be aware of the life threatening complications of pregnancy and labour.

\section{Further Reading}

Managing Obstetric Emergencies

C Cox and K Grady Bios Scientific 1999 